\title{
GENERALIZED BALANCED TOURNAMENT DESIGNS
}

\author{
E. R. LAMKEN
}

\begin{abstract}
A generalized balanced tournament design, $\operatorname{GBT} D(n, k)$, defined on a $k n$-set $V$, is an arrangement of the blocks of a $(k n, k, k-1)$ $B I B D$ defined on $V$ into an $n \times(k n-1)$ array such that (1) every element of $V$ is contained in precisely one cell of each column, and (2) every element of $V$ is contained in at most $k$ cells of each row. In this paper, we introduce $\operatorname{GBTD}(n, k) s$ and describe connections between these designs and several other types of combinatorial designs. We also show how to use GBTDS to construct resolvable, near resolvable, doubly resolvable and doubly near resolvable $B I B D s$.
\end{abstract}

\section{INTRODUCTION}

A generalized balanced tournament design, $G B T D(n, k)$, defined on a $k n$ set $V$, is an arrangement of the blocks of a $(k n, k, k-1)$-BIBD defined on $V$ into an $n \times(k n-1)$ array such that

(1) every element of $V$ is contained in precisely one cell of each column, and

(2) every element of $V$ is contained in at most $k$ cells of each row.

This generalizes the idea of a balanced tournament design and we note that a $\operatorname{GBTD}(n, 2)$ is a $B T D(n),[32]$.

A simple but very important observation is stated in the next lemma.

Lemma 1.1. Every element of a $G B T D(n, k)$ is contained $k$ times in $(n-1)$ rows and $(k-1)$ times in the remaining row.

Let $G$ be a $G B T D(n, k)$. An element which is contained in only $k-1$ cells of row $i$ of $G$ is called a deficient element of row $i$. It is easily seen that each row of $G$ contains exactly $k$ deficient elements. These elements are called the deficient $k$-tuple of row $i$. The deficient elements of row $i$ need not occur in a common block of row $i$.

Lemma 1.2. The deficient $k$-tuples of $G$ partition the set $V$ into $n k$-tuples.

We illustrate these definitions by displaying a $\operatorname{GBTD}(3,3)$ in Figure 1 . The

Received by the editors November 22, 1988.

1980 Mathematics Subject Classification (1985 Revision). Primary 05B05, 05B15, 05B30.

This research was supported by a Postdoctoral membership at the Institute for Mathematics and its Applications, University of Minnesota, Minneapolis. 
deficient triples of rows 1,2 and 3 are respectively $\{4,6,8\},\{1,2,9\}$ and $\{3,5,7\}$.

Let $C=\left(C_{1}, C_{2}, \ldots, C_{n}\right)^{T}$ where $C_{i}, 1 \leq i \leq n$, is the deficient $k$-tuple of row $i$ of $G$. If $C$ occurs as a column in $G k-1$ times, $G$ is said to have property $C$. The $G B T D(3,3)$ displayed in Figure 1 does not have property $C$. A $G B T D(4,3)$ with Property $C$ is displayed in Figure 2.

\begin{tabular}{|llllllll|}
\hline 129 & 349 & 569 & 145 & 357 & 178 & 238 & 267 \\
357 & 167 & 138 & 236 & 468 & 245 & 749 & 589 \\
468 & 258 & 247 & 789 & 129 & 369 & 165 & 134 \\
\hline
\end{tabular}

Figure 1. A $G B T D(3,3)$

\begin{tabular}{|lllllllllll|}
\hline$F G J$ & $E I L$ & $D H K$ & $F H L$ & $E G K$ & $D I J$ & $F I K$ & $D G L$ & $E H J$ & $A B C$ & $A B C$ \\
$A I L$ & $C H K$ & $C I J$ & $B G J$ & $B H L$ & $A G K$ & $A H J$ & $B I K$ & $C G L$ & $D E F$ & $D E F$ \\
$B E K$ & $B F J$ & $A E L$ & $C D K$ & $A D J$ & $C F L$ & $B D L$ & $C E F$ & $A F K$ & $G H I$ & $G H I$ \\
$C D H$ & $A D G$ & $B F G$ & $A E I$ & $C F I$ & $B E H$ & $C E G$ & $A F H$ & $B D I$ & $J K L$ & $J K L$ \\
\hline
\end{tabular}

Figure 2. A $G B T D(4,3)$ with Property $C$

A near generalized balanced tournament design, $N G B T D(n, k)$, defined on a $(k n+1)$-set $V$, is an arrangement of the blocks of a $(k n+1, k, k-1)-B I B D$ defined on $V$ into an $n \times(k n+1)$ array so that

(1) every element of $V$ occurs precisely $k$ times in each row;

(2) every column of the array contains $k n$ distinct elements of $V$, and

(3) the columns form a near resolution of the $(k n+1, k, k-1)-B I B D$.

A near generalized balanced tournament design is a generalization of an odd balanced tournament design; in particular, a $\operatorname{NGBTD}(n, 2)$ is an $O B T D(n)$, [19]. We illustrate this definition by displaying a $\operatorname{NGBTD}(2,3)$ in Figure 3.

\begin{tabular}{|lllllll|}
\hline 124 & 235 & 346 & 450 & 561 & 602 & 013 \\
356 & 460 & 501 & 612 & 023 & 134 & 245 \\
\hline
\end{tabular}

Figure 3. A $N G B T D(2,3)$

The existence of $B T D(n) s$ or $G B T D(n, 2) s$ was established in 1977 [32]; there exists a $G B T D(n, 2)$ for $n$ a positive integer, $n \neq 2$. The existence of $O B T D(n) s$ or $N G B T D(n, 2) s$ is also well known and easy to prove [21]; there exists a $N G B T D(n, 2)$ for $n$ a positive integer. Factored $B T D s$, partitioned $B T D s, B T D s$ with almost orthogonal resolutions and $O B T D s$ with orthogonal resolutions have been investigated extensively in the past few years. Results on these designs can be found in the following papers $[15,16,17,18,8,20$, 
21, 22]. Most of these designs have become of interest recently because of their relationships to other types of combinatorial designs. Of particular interest to us are the connections between balanced tournament designs and resolvable and doubly resolvable $B I B D s[19]$.

The purpose of this paper is to introduce generalized balanced tournament designs including factored and partitioned GBTDs, as well as near generalized balanced tournament designs. We will show that these designs are also of considerable interest because of their relationships to other designs. One of our main results will be to show that $\operatorname{PGBTD}(n, k) s$ can be used directly to construct $D R(n k, k, k-1)-B I B D s$. This will provide a surprisingly easy way to construct the doubly resolvable designs. This paper is the first of a series of papers on generalized balanced tournament designs $[10,11]$. The remaining papers describe in detail constructions for GBTDs and existence results for these designs. As we noted above and in [20], existence results for $\operatorname{GBT} D(n, k)$ s with $k=2$ are known. In [11], we consider the next case, $k=3$. For completeness, we list two of the existence results here.

Theorem 1.3 [11]. For $n$ a positive integer, $n \geq 3$, there exists a $\operatorname{GBTD}(n, 3)$ except possibly for $n \in\{14,17,23,47,62,68,95,102,115\}$.

Theorem 1.4 [11]. For $n$ a positive integer, there exists a $\operatorname{NGBTD}(n, 3)$ except possibly for $n \in\{3,38,39,118\}$.

In the next section, we collect some preliminary definitions and results on resolvable, near resolvable, doubly resolvable and doubly near resolvable $B I B D S$. In $\S 3$, we define and discuss factored GBTDs (FGBTDs); we will show that $F G B T D s$ with Property $C$ can be used to construct 1-dimensional frames. Partitioned GBTDs (PGBTDs) are defined in $\S 4$ and the equivalence of these designs to doubly resolvable designs is described. In $\S 5$, we generalize several constructions from [19] which use GBTDs and $N G B T D s$ to construct resolvable, near resolvable, doubly resolvable and doubly near resolvable $B I B D S$.

\section{Preliminary Definitions AND Results}

Generalized balanced tournament designs can be used to construct resolvable, near resolvable, doubly resolvable and doubly near resolvable balanced incomplete block designs. In this section, we collect some of the necessary definitions and existence results for these constructions.

A balanced incomplete block design $(B I B D) D$ is a collection $B$ of subsets (blocks) taken from a finite $v$-set $V$ of elements with the following properties:

(1) Every pair of distinct elements from $V$ is contained in precisely $\lambda$ blocks of $B$.

(2) Every block contains exactly $k$ elements.

We denote such a design as a $(v, k, \lambda)-B I B D$. It is well known that necessary conditions for the existence of a $(v, k, \lambda)-B I B D$ are

$$
\lambda(v-1) \equiv 0 \quad(\bmod (k-1)) \quad \text { and } \quad \lambda v(v-1) \equiv 0 \quad(\bmod k(k-1)) .
$$


A $(v, k, \lambda)-B I B D \quad D$ is said to be resolvable (and denoted by $(v, k, \lambda)$ $R B I B D)$ if the blocks of $D$ can be partitioned into classes $R_{1}, R_{2}, \ldots, R_{r}$ (resolution classes) where $r=\frac{\lambda(v-1)}{(k-1)}$ such that each element of $D$ is contained in precisely one block of each class. The classes $R_{1}, R_{2}, \ldots, R_{r}$ form a resolution of $D$. Necessary conditions for the existence of a $(v, k, \lambda)-R B I B D$ are that $(1)$ be satisfied and that $v \equiv 0(\bmod k)$.

A $(v, k, \lambda)-B I B D D$ is said to be near resolvable (and denoted by $N R(v, k$,

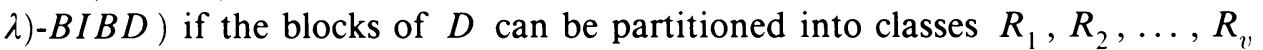
(resolution classes) such that for each element $x$ of $D$ there is precisely one class which does not contain $x$ in any of its blocks and each class contains precisely $v-1$ distinct elements of the design. Necessary conditions for the existence of $N R(v, k, \lambda)-B I B D s$ are $v \equiv 1(\bmod k)$ and $\lambda=k-1$.

In general, the spectrum of resolvable and near resolvable $(v, k, \lambda)-B I B D s$ remains open. The existence of these designs has been established for several small values of $k$ and $\lambda$. In this paper, we are primarily interested in the cases $\lambda=k-1$. For completeness, we include some of the results for other values of $\lambda$. It is well known that there exists a $(v, 2,1)-R B I B D$ for $v$ a positive integer and $v \equiv 0(\bmod 2)$ and that there exists a $N R(v, 2,1)-B I B D$ for $v$ a positive integer and $v \equiv 1(\bmod 2)$. The existence of $R B I B D s$ and $N R B I B D s$ has also been established for block size $k=3$; the necessary conditions are also sufficient (with one exception).

Theorem 2.1 (Ray-Chaudhuri and Wilson [28]). There exists $a(v, 3,1)-R B I B D$ if and only if $v \equiv 3(\bmod 6)$.

Theorem 2.2 (Hanani [6]). (i) There exists $a(v, 3,2)-R B I B D$ if and only if $v \equiv 0(\bmod 3)$ and $v \neq 6$. (ii) There exists a $N R(v, 3,2)-B I B D$ if and only if $v \equiv 1(\bmod 3), v \geq 4$.

For block size $k=4$, the following results are known.

Theorem 2.3 (Hanani, Ray-Chaudhuri and Wilson [7]). There exists $a(v, 4,1)$ $R B I B D$ if and only if $v \equiv 4(\bmod 12)$.

Theorem 2.4 (Baker [1]). There exists $a(v, 4,3)-R B I B D$ if and only if $v \equiv$ $0(\bmod 4)$.

For block size larger than 4 , very little is known. Recent results for $(v, 5,1)$ $R B I B D s$ can be found in [2], and results for $(v, 6,10)-R B I B D s$ appear in [1]. For the designs that we are interested in with $\lambda=k-1$, the next case where some results have been obtained is $k=8$.

Theorem 2.5 (Rokowska [31]). For all $v \equiv 0(\bmod 8)$ which are not divisible by 3,5 and 7 , there exists $a(v, 8,7)-R B I B D$.

Some other infinite classes of resolvable $B I B D s$ are known to exist. We include some existence results for the case $\lambda=k-1$ in our list of results for doubly resolvable $B I B D S$. 
A $(v, k, \lambda)-B I B D$ is said to be doubly (near) resolvable if there exist two (near) resolutions $R$ and $R^{\prime}$ of the blocks such that $\left|R_{i} \cap R_{j}^{\prime}\right| \leq 1$ for all $R_{i} \in$ $R, R_{j}^{\prime} \in R^{\prime}$. (It should be noted that the blocks of the design are considered as being labelled so that if a subset of the elements occurs as a block more then once the blocks are treated as distinct.) The (near) resolutions $R$ and $R^{\prime}$ are called orthogonal resolutions of the design. A doubly resolvable $(v, k, \lambda)-B I B D$ is denoted by $D R(v, k, \lambda)-B I B D$ and a doubly near resolvable $(v, k, \lambda)-B I B D$ by $D N R(v, k, \lambda)-B I B D$.

A Kirkman square with block size $k$, order $v$ and index $\lambda, K S_{k}(v ; 1, \lambda)$, is a $t \times t$ array $K(t=\lambda(v-1) /(k-1))$ defined on a $v$-set $V$ such that

(1) each element of $V$ is contained in precisely one cell of each row and column of $K$;

(2) each cell of $K$ is either empty or contains a $k$ subset of $V$;

(3) The collection of blocks obtained from the nonempty cells of $K$ is a $(v, k, \lambda)-B I B D$.

We can use a pair of orthogonal resolutions of a $D R(v, k, \lambda)-B I B D$ to construct a $K S_{k}(v ; 1, \lambda)$. The rows of the array form one resolution of the $D R(v, k, \lambda)-B I B D$ and the columns form an orthogonal resolution. Similarly, we can use a pair of orthogonal resolutions of a $D N R(v, k, \lambda)-B I B D$ to construct a $v \times v$ array. The rows of the array will form one resolution of the design and the columns will form an orthogonal resolution. If the $\operatorname{DNR}(v, k, \lambda)$ $B I B D$ has the additional property that under an appropriate ordering of the resolution classes $R$ and $R^{\prime}, R_{i} \cup R_{i}^{\prime}$ contains precisely $v-1$ distinct elements of the design for all $i$, then the array is called a $(1, \lambda ; k, v, 1)$-frame [24]. Note that the diagonal of a $(1, \lambda ; k, v, 1)$-frame is empty and a unique element of the design can be associated with each cell $(i, i)$. (This distinction between $(1, \lambda ; k, v, 1)$-frames and $D N R(v, k, \lambda)-B I B D s$ is important in recursive constructions.)

Even less is known about the spectrum of doubly resolvable and doubly near resolvable $(v, k, \lambda)-B I B D s$. Although several infinite classes of $D R(v, k, \lambda)$ $B I B D s$ are known for $k \geq 3,[5,3,38]$, the existence of $D R(v, k, \lambda)-B I B D s$ has been settled only for $k=2$ and $\lambda=1$. $(D R(v, 2,1)-B I B D s$ are also called Room squares.)

Theorem 2.6 (Mullin and Wallis [27]). There exists a $D R(v, 2,1)-B I B D$ for $v$ a positive integer, $v \equiv 0(\bmod 2)$ and $v \neq 4$ or 6 .

An immediate corollary of this result is the following.

Corollary 2.7. There exists a $\operatorname{DNR}(v, 2,1)-B I B D$ (and $a(1,1 ; 2, v, 1)$-frame) for $v \equiv 1(\bmod 2), v \neq 3$ or 5 .

For block size $k=3$ the problem is more difficult. The best result, thus far, for $\lambda=1$ is asymptotic.

Theorem 2.8 (Rosa and Vanstone [30]). There exists a constant $v_{1}$ such that for all $v_{1} \leq v$ and $v \equiv 3(\bmod 6)$ there exists a $D R(v, 3,1)-B I B D$. 
The problem is more tractable for $k=3$ and $\lambda=2$. The best results, thus far, for $D N R(v, 3,2)-B I B D s$ are the following.

Theorem 2.9 (Lamken and Vanstone [24]). For $n$ a positive integer, there exists a $(1,2 ; 3,15 n+1,1)$-frame or a $D N R(15 n+1,3,2)$-BIBD.

Theorem 2.10 (Lamken [13]). There exists a $D N R(v, 3,2)-B I B D$ for $v \equiv 1$ $(\bmod 9)$ except possibly for $v=55$.

There are two analogous results for $D R(v, 3,2)-B I B D s$.

Theorem 2.11 (Lamken and Vanstone [23]). For $v \equiv 3(\bmod 12)$, there exists $a$ $D R(v, 3,2)-B I B D$ which contains as a subarray a $D R(3,3,2)-B I B D$.

Theorem 2.12 (Lamken [13]). Let $M=\{6,8,10,12,14,15,18,20,22,24$, $26,30,32,34,38,39,42,44,46,48,51,52,58,118\}$, and let $v=9 m+3$ where $m$ is a positive integer, $m \notin M$. Then there exists a $D R(v, 3,2)$ $B I B D$.

Quite recently, the spectrum of $D R(v, 3,2)-B I B D s$ has been determined with a small number of possible exceptions, [12]. We note some of the ideas used for this result in $\S 4$.

For $k \geq 3$, some infinite classes of $D R(v, k, \lambda)-B I B D s$ have been constructed. We include two of these results.

Theorem 2.13 (Fuji-Hara and Vanstone [5]). For $p$ a prime power and $n a$ positive integer greater than 2 , there exists a $D R\left(p^{n}, p, 1\right)-B I B D$.

Theorem 2.14 (Curran and Vanstone [3]). For $k$ a prime power and $k \geq 3$ there exists a $D R\left(k^{2}+k, k, k-1\right)-B I B D$.

Finally, we note that the generalization of the Kirkman square has been studied in $[14,25,26]$. Frames have also been investigated in a more general setting; we refer to $[4,24,37,38,36]$ for some of these results.

\section{FACTORED GENERALIZED BALANCED TOURNAMENT DESIGNS}

Let $G$ be a $G B T D(n, k)$ defined on $V$, and let $C=\left(C_{1}, C_{2}, \ldots, C_{n}\right)^{T}$ where $C_{i}, 1 \leq i \leq n$, be the deficient $k$-tuple of row $i$ of $G$. Suppose the blocks in row $i$ of $G \cup C_{i}$ can be partitioned into $k$ sets of $n$ blocks each, $F_{i 1}, F_{i 2}, \ldots, F_{i k}$, so that every element of $V$ occurs precisely once in $F_{i j}$ for $j=1,2, \ldots, k$. If every row of $G$ has this property, then $G$ is called a factored generalized balanced tournament design and is denoted by $F G B T D(n, k)$. We will call $F_{i 1}, F_{i 2}, \ldots, F_{i(k-1)}, F_{i k}$ the factors of row $i$. The $G B T D(4,3)$ displayed in Figure 2 is factored; the factors are listed in Table 1. We note that if a $F G B T D(n, k)$ also has Property $C$ then $C_{i} \in F_{i j}$ for $j=1,2, \ldots, k$ and $i=1,2, \ldots, n$.

The existence of factored generalized balanced tournament designs with Property $C$ is equivalent to the existence of 1 -dimensional frames with or- 
thogonal resolutions. We will need some additional definitions and notation in order to describe these frames.

TABLE 1

Factors for the $F G B T D(4,3)$ displayed in Figure 2

\begin{tabular}{|lcccc|}
\hline Row 1: & $A B C$ & $F G J$ & $E I L$ & $D H K$ \\
& $A B C$ & $F H L$ & $E G K$ & $D I J$ \\
& $A B C$ & $F I K$ & $D G L$ & $E H J$ \\
Row 2: & $D E F$ & $B I K$ & $A H J$ & $C G L$ \\
& $D E F$ & $A I L$ & $C H K$ & $B G J$ \\
& $D E F$ & $C I J$ & $B H L$ & $A G K$ \\
& & & & \\
Row 3: & $G H I$ & $B E K$ & $A D J$ & $C F L$ \\
& $G H I$ & $B F J$ & $A E L$ & $C D K$ \\
& $G H I$ & $B D L$ & $C E J$ & $A F K$ \\
& & & & \\
Row 4: & $J K L$ & $C D H$ & $B F G$ & $A E I$ \\
& $J K L$ & $A F H$ & $C E G$ & $B D I$ \\
& $J K L$ & $B E H$ & $C F I$ & $A D G$ \\
\hline
\end{tabular}

A group divisible design (GDD) is a collection $B$ of subsets (blocks) of size $k$ taken from a $v$-set $V$ along with a partition of $V$ in groups $G_{1}, G_{2}, \ldots, G_{n}$ such that

(1) any two elements from distinct groups are contained in precisely $\lambda_{2}$ blocks of $B$; and

(2) any two distinct elements from the same groups are contained in precisely $\lambda_{1}$ blocks of $B \quad\left(\lambda_{1}<\lambda_{2}\right)$.

We denote such a design by $G D D\left(v ; k ; G_{1}, G_{2}, \ldots, G_{n} ; \lambda_{1}, \lambda_{2}\right)$. If $\left|G_{i}\right|=h$ for $i=1,2, \ldots, n$, we denote the design by $G D D\left(v ; k ; h ; \lambda_{1}, \lambda_{2}\right)$.

A GDD is resolvable if the blocks can be partitioned into classes (resolution classes) $R_{1}, R_{2}, \ldots, R_{r}$ such that every element of $V$ is contained in precisely one block of each $R_{i}, \quad 1 \leq i \leq r$. The collection of resolution classes is called a resolution of the GDD.

Lemma 3.1 (Vanstone [38]). In a resolvable GDD all groups have the same size. We denote a resolvable GDD with $\left|G_{i}\right|=h$ for all $i$ by $R G D D\left(v ; k ; h ; \lambda_{1}, \lambda_{2}\right)$. Let $V$ be a set of $n k$ elements. Let $G_{1}, G_{2}, \ldots, G_{n}$ be a partition of $V$ into $n$ sets where $\left|G_{i}\right|=k$ for $i=1,2, \ldots, n$. A 1 -dimensional $\left\{G_{1}, G_{2}, \ldots, G_{n}\right\}$ frame $F$ with block size $k$, index $k-1$ and group size $k$ is a set $P$ of partial resolution classes of $V$ which satisfies the following properties. Let $P=\left\{P_{i 1}, P_{i 2}, \ldots, P_{i k} \mid i=1,2, \ldots, n\right\}$.

(1) Each partial resolution class $P_{i j}$ contains $n-1$ blocks of size $k$. 
(2) Every element of $V-G_{i}$ occurs precisely once in $P_{i j}$ for $j=1,2$, $\ldots, n$.

(3) The collection of blocks in $P$ is a $G D D\left(n k ; k ; G_{1}, \ldots, G_{n} ; 0, k-1\right)$. We will call $F$ a 1-dimensional $(1, k-1 ; k, n, k)$-frame. (This notation is consistent with our notation for frames in general [24].) A 1-dimensional $(1,2 ; 3,4,3)$-frame is displayed in Figure 4 to illustrate this definition. We note that 1-dimensional frames have become of interest in the past few years and have been used to prove several new results for resolvable designs [29, 35, 36].

Let $G$ be a $R G D D\left(n k ; k ; G_{1}, G_{2}, \ldots, G_{n} ; 0, k-1\right)$ defined on a set $V$. Let $R_{1}, R_{2}, \ldots, R_{(n-1) k-2}$ denote the resolution classes of a resolution $R$ of $G$. Suppose there exists a 1 -dimensional $(1, k-1 ; k, n, k)$-frame $F$ where $F$ is a $\left\{G_{1}, G_{2}, \ldots, G_{n}\right\}$-frame and $F$ contains the blocks of $G$. Let $P=$ $\left\{P_{i 1}, P_{i 2}, \ldots, P_{i k} \mid i=1,2, \ldots, n\right\}$ denote the partial resolution classes of $F$. If $\left|R_{i} \cap\left(P_{j 1} \cup P_{j 2} \cup \cdots \cup P_{j k}\right)\right|=1$ for $j=1,2, \ldots, n$ and $i=1,2, \ldots, n k-$ $k-2$, then we say $R$ is an orthogonal resolution for $F$. As an example of this definition, the first nine columns of the $\operatorname{FGBTD}(4,3)$ in Figure 2 form an orthogonal resolution for the 1-dimensional frame in Figure 4.

\begin{tabular}{|llll|}
\hline$P_{11}$ & $F G J$ & $E I L$ & $D H K$ \\
$P_{12}$ & $F H L$ & $E G K$ & $D I J$ \\
$P_{13}$ & $F I K$ & $D G L$ & $E H J$ \\
& & & \\
$P_{21}$ & $B I K$ & $A H J$ & $C G L$ \\
$P_{22}$ & $A I L$ & $C H K$ & $B G J$ \\
$P_{23}$ & $C I J$ & $B H L$ & $A G K$ \\
& & & \\
$P_{31}$ & $B E K$ & $A D J$ & $C F L$ \\
$P_{32}$ & $B F J$ & $A E L$ & $C D K$ \\
$P_{33}$ & $B D L$ & $C E J$ & $A F K$ \\
& & & \\
$P_{41}$ & $C D H$ & $B F G$ & $A E I$ \\
$P_{42}$ & $A F H$ & $C E G$ & $B D I$ \\
$P_{43}$ & $B E H$ & $C F I$ & $A D G$ \\
\hline
\end{tabular}

FIGURE 4. A 1-dimensional $(1,2 ; 3,4,3)$-frame

We can now prove the following equivalence.

Theorem 3.2. There exists a $F G B T D(n, k)$ with Property $C$ if and only if there exists a 1-dimensional $(1, k-1 ; k, n, k)$-frame $F$ with an orthogonal resolution.

Proof. Let $G$ be a $F G B T D(n, k)$ with Property $C$ defined on a set $V$ of $n k$ elements. Suppose the factors of row $i$ are $F_{i 1}, F_{i 2}, \ldots, F_{i(k-1)}, F_{i k}$ for $i=1,2, \ldots, n$. Since $G$ has Property $C, C_{i} \in F_{i j}$ for $j=1,2, \ldots, k-1$. 
Let $F_{i j}^{\prime}=F_{i j}-C_{i}$ for $j=1,2, \ldots, k-1$ and let $F_{i k}^{\prime}=F_{i k}$. The collection of blocks in $\bigcup_{i=1,2, \ldots, n ; j=1,2, \ldots k} F_{i j}^{\prime}$ is a $G D D(n k ; k ; k ; 0, k-1)$. The groups of the design are the deficient $k$-tuples, $C_{1}, C_{2}, \ldots, C_{n}$. Every element in $V-C_{i}$ occurs precisely once in the blocks of $F_{i j}^{\prime}, j=1,2, \ldots, k$. We can construct a 1 -dimensional $(1, k-1 ; k, n, k)$-frame $F$ by defining the partial resolution classes as follows: $P_{i j}=F_{i j}^{\prime}$ for $j=1,2, \ldots, k$ and $i=$ $1,2, \ldots, n$. If we delete the $k-1$ copies of $C$ from $G$, it is clear that the remaining columns of $G$ form resolution classes for a resolution $R$ of the resulting $G D D(n k ; k ; k ; 0, k-1)$. Since each resolution class contains precisely one block from each row of the $\operatorname{FGBTD}(n, k)$ and thus precisely one block from $\left(P_{j 1} \cup P_{j 2} \cup \cdots \cup P_{j k}\right)$, the resolution $R$ is an orthogonal resolution for the 1-dimensional frame $F$.

This construction can be reversed. If we start with a 1-dimensional $(1, k-1$; $k, n, k)$-frame with an orthogonal resolution, we can construct a $F G B T D(n, k)$ as follows. Let $F$ be a $\left\{C_{1}, C_{2}, \ldots, C_{n}\right\}$-frame defined on an $n k$ set $V$. The blocks in row $i$ of the $F G B T D(n, k)$ will be the blocks in $P_{i 1} \cup P_{i 2} \cup \cdots \cup P_{i k}$ together with $k-1$ copies of the group $C_{i}$. The $i$ th resolution class of the orthogonal resolution $R, R_{i}$, will contain the blocks of the $i$ th column of the $F G B T D(n, k)$ for $i=1,2, \ldots, n k-k-2$. Finally, the $\operatorname{FGBTD}(n, k)$ will contain $k-1$ copies of the column $C=\left(C_{1}, C_{2}, \ldots, C_{n}\right)^{T}$.

Corollary 3.3. If there exists a $F G B T D(n, k)$ with Property $C$, then there exists $a(1, k-1 ; k, n, k)$-frame.

The spectrum of $F G B T D(n, k)$ with Property $C$ has been determined for $k=2$ and for $k=3$ with a finite number of possible exceptions.

Theorem 3.3 [15]. For $n$ a positive integer, $n \neq 2$ or 4 , there exists a FGBTD $(n, 2)$ with Property $C$.

Theorem 3.4 [11]. For $n$ a positive integer, $n \geq 4$, there exists a $F G B T D(n, 3)$ with Property $C$ except possibly for $n \in\{14,15,17,18,19,23,27,31,39$, $47,62,68,87,102,103,114,115,127,131,151,159\}$.

\section{PARTitioned generalized BALANCED TOURNAMENT DESignS}

Let $G$ be a $G B T D(n, k)$ defined on $V$. Suppose we can partition the columns of $G$ into $k+1$ sets $B_{1}, B_{2}, \ldots, B_{k+1}$ where $\left|B_{i}\right|=n$ for $i=$ $1,2, \ldots, k-2,\left|B_{i}\right|=n-1$ for $i=k-1, k$ and $\left|B_{k+1}\right|=1$ such that

(1) every element of $V$ occurs precisely once in each row and column of $B_{i}$ for $i=1,2, \ldots, k-2$, and

(2) every element of $V$ occurs precisely once in each row and column of $B_{i} \cup B_{k+1}$ for $i=k-1$ and $i=k$.

Then the $\operatorname{GBTD}(n, k), G$, is called partitioned and we denote the design by $\operatorname{PGBTD}(n, k)$. Let $C=\left(C_{1}, C_{2}, \ldots, C_{n}\right)^{T}$ where $C_{i}, 1 \leq i \leq n$, is the deficient $k$-tuple of row $i$ of $G$. It is clear that if $G$ is partitioned then 


\begin{tabular}{|l|l|l|l|l|l|l|l|}
\hline 013 & 91318 & 14209 & $\beta 168$ & $\alpha 172$ & 71011 & $\gamma 615$ & 51219 \\
\hline 81419 & $\beta 102$ & $\alpha 1117$ & 145 & $\gamma 09$ & 151618 & 3712 & 61320 \\
\hline$\alpha 511$ & 161920 & $\gamma 153$ & 91012 & 1816 & 3813 & $\beta 417$ & 7140 \\
\hline$\gamma 918$ & 346 & 12160 & 1727 & $\beta 1911$ & $\alpha 205$ & 101314 & 8151 \\
\hline 61015 & 11171 & $\beta 135$ & $\alpha 1420$ & 478 & $\gamma 312$ & 18190 & 9162 \\
\hline$\beta 720$ & $\alpha 814$ & 1912 & $\gamma 186$ & 121315 & 049 & 61116 & 10173 \\
\hline 131617 & $\gamma 120$ & 679 & 15193 & 20510 & $\beta 114$ & $\alpha 28$ & 11184 \\
\hline 2412 & 5715 & 81018 & 11130 & 14163 & 17196 & 2019 & $\alpha \beta \gamma$ \\
\hline
\end{tabular}

$B_{1}$

\begin{tabular}{|l|l|l|l|l|l|l|}
\hline 141718 & $\gamma 131$ & 7810 & 16204 & 0611 & $\beta 215$ & $\alpha 39$ \\
\hline 124 & 101419 & 1505 & $\beta 179$ & $\alpha 183$ & 81112 & $\gamma 716$ \\
\hline 91520 & $\beta 113$ & $\alpha 1218$ & 256 & $\gamma 110$ & 161719 & 4813 \\
\hline$\alpha 612$ & 17200 & $\gamma 164$ & 101113 & 1927 & 3914 & $\beta 518$ \\
\hline$\gamma 1019$ & 457 & 13171 & 1838 & $\beta 2012$ & $\alpha 06$ & 111415 \\
\hline 71116 & 12182 & $\beta 146$ & $\alpha 150$ & 589 & $\gamma 413$ & 19201 \\
\hline$\beta 80$ & $\alpha 915$ & 2023 & $\gamma 197$ & 131416 & 1510 & 61217 \\
\hline 3513 & 6816 & 91119 & 12141 & 15174 & 18207 & 0210 \\
\hline
\end{tabular}

$B_{2}$

\begin{tabular}{|l|l|l|l|l|l|l|l|}
\hline$\beta 91$ & $\alpha 1016$ & 034 & $\gamma 208$ & 141517 & 2611 & 71318 & 51219 \\
\hline 151819 & $\gamma 142$ & 8911 & 1705 & 1712 & $\beta 316$ & 2410 & 61320 \\
\hline 235 & 111520 & 1616 & $\beta 1810$ & $\alpha 194$ & 91213 & $\gamma 817$ & 7140 \\
\hline 10160 & $\beta 124$ & $\alpha 1319$ & 367 & $\gamma 211$ & 171820 & 5914 & 8151 \\
\hline$\alpha 713$ & 1801 & $\gamma 175$ & 111214 & 2038 & 41015 & $\beta 619$ & 9162 \\
\hline$\gamma 1120$ & 568 & 14182 & 1949 & $\beta 013$ & $\alpha 17$ & 121516 & 10173 \\
\hline 81217 & 13193 & $\beta 157$ & $\alpha 161$ & 6910 & $\gamma 514$ & 2002 & 11184 \\
\hline 4614 & 7917 & 101220 & 13152 & 16185 & 1908 & 1311 & $\alpha \beta \gamma$ \\
\hline
\end{tabular}

Figure 5. $P=\left[\begin{array}{llll}B_{1} & B_{2} & B_{3} & B_{4}\end{array}\right]$

$B_{k+1}=C$. If $G$ is partitioned and has Property $C$, then each of the $n \times n$ arrays $B_{1}, B_{2}, \ldots, B_{k-2}$ will contain a copy of $C$ as a column. We illustrate this definition by displaying a $\operatorname{PGBTD}(8,3)$ in Figure 5 . This design also has Property $C$.

The existence of $P G B T D s$ is equivalent to the existence of Kirkman squares in diagonal form. Let $K$ be a $K S_{k}(n k ; 1, k-1)$ defined on $V . K$ is said to be in diagonal form if it can be written in the following form.

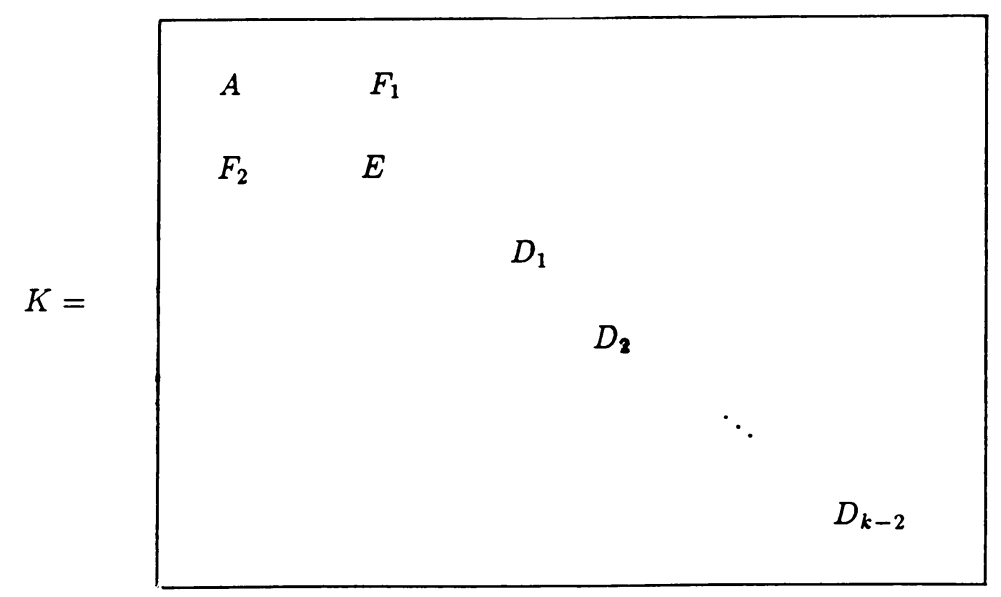




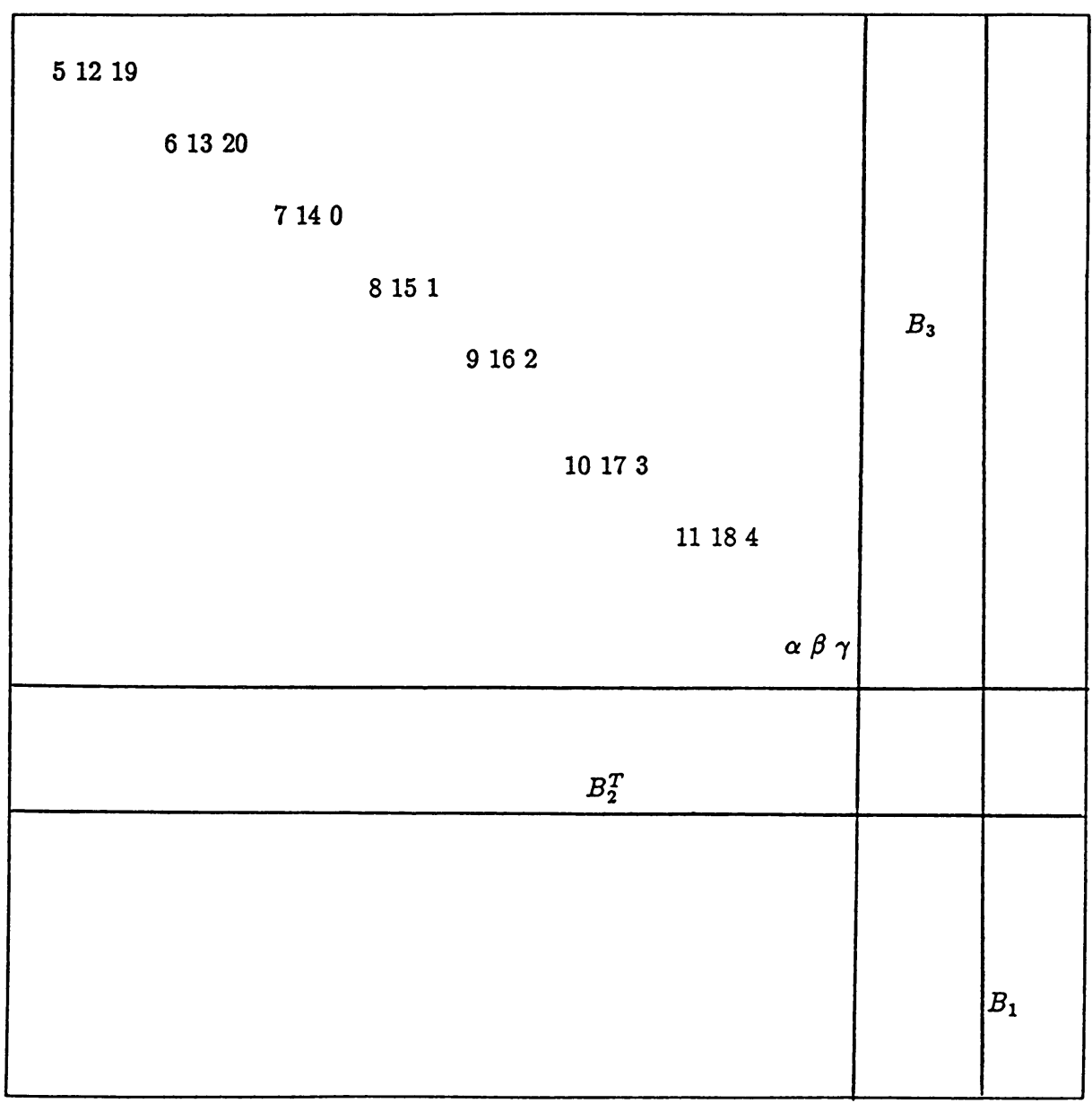

FiguRE 6. A $K S_{3}(24 ; 1,2)\left(B_{1}, B_{2}, B_{3}\right.$ are displayed in Figure 5)

$A$ is an $n \times n$ array which contains $n \quad k$-tuples along its diagonal. $F_{1}$ is an $n \times n-1$ array of $k$-tuples; $F_{2}$ is an $n-1 \times n$ array of $k$-tuples; and $E$ is an $n-1 \times n-1$ empty array. Each of the arrays $D_{i}, 1 \leq i \leq k-2$, is an $n \times n$ array of $k$-tuples. All of the remaining cells of $K$ are empty. We recall that $K$ displays a pair of orthogonal resolutions for a $D R(v, k, k-1)-B I B D$.

Theorem 4.1. There exists a $\operatorname{PGBTD}(n, k)$ if and only if there exists a $K S_{k}(n k$; $1, k-1)$ in diagonal form.

Proof. Let $P$ be a $\operatorname{PGBTD}(n, k)$ defined on $V$. Let $B_{1}, B_{2}, \ldots, B_{k+1}$ be a corresponding partitioning of the columns of $P$. Let $C=\left(C_{1}, C_{2}, \ldots, C_{n}\right)^{T}$ where $C_{i}, 1 \leq i \leq n$, is the deficient $k$-tuple of row $i$ of $P$. Recall that $B_{k+1}=C$. We now define the arrays $A, F_{1}, F_{2}, D_{1}, D_{2}, \ldots, D_{k-2}$ which provide the diagonal form of a $K S_{k}(n k ; 1, k-1), K$, defined on $V . A$ is an $n \times n$ array which contains $C_{i}$ in cell $(i, i)$. (All of the other cells of 
$A$ are empty.) Let $F_{1}=B_{k}$ and let $F_{2}=B_{k-1}^{T}$. Finally, let $D_{i}=B_{i}$ for $i=1,2, \ldots, k-2$. It is easy to verify that we have constructed the arrays for a $K S_{k}(n k ; 1, k-1)$ in diagonal form.

The construction can be reversed. Let $K$ be a $K S_{k}(n k ; 1, k-1)$ defined on $V$. Let $C=B_{k+1}=\left(C_{1}, C_{2}, \ldots, C_{n}\right)^{T}$ where $C_{i}$ is the $k$-tuple contained in cell $(i, i)$ of $A$. It is straightforward to verify that $\left[D_{1} D_{2} \cdots D_{k-2} F_{2}^{T} F_{1} C\right]$ is a $\operatorname{PGBTD}(n, k)$ defined on $V$.

A $K S_{3}(24 ; 1,2)$ in diagonal form which is equivalent to the $\operatorname{PGBTD}(8,3)$ in Figure 5 is displayed in Figure 6.

We note that for $k=2$ this construction reduces to the construction used by D. R. Stinson in [34] to provide the connection between Room squares and PBTDs .

Corollary 4.2 (D. R. Stinson [34]). There exists a PGBTD(n,2) if and only if there exists a $\operatorname{MESRS}(2 n-1)$.

The following result has been established for $k=2$.

Theorem 4.3 (Lamken and Vanstone [16], [17], [18], Lamken [8]). There exists a $\operatorname{PGBTD}(n, 2)$ for all $n \geq 5$ except possibly for $n \in\{9,11,15,26,28,34,44\}$.

Constructions and existence results for $\operatorname{PGBTD}(n, 3) s$ have been used quite recently to determine the spectrum of $D R(3 n, 3,2)-B I B D s$ or $K S_{3}(3 n ; 1,2) s$, [12]. There are now only a small number of possible exceptions for the existence of $K S_{3}(3 n ; 1,2) s$; these results are discussed in detail in [12]. We refer to $[10,11,12]$ for constructions and results for $\operatorname{PGBTD}(n, k) s$ with $k \geq 3$.

\section{Using GBTDS to CONSTRUCT RESOLVABLE $B I B D S$}

In this section, we generalize the constructions in [19] to use generalized balanced tournament designs to construct resolvable, near resolvable, doubly resolvable and doubly near resolvable $B I B D s$. The first three constructions are for resolvable and near resolvable designs.

Theorem 5.1. If there exists a GBTD $(n, k), a(k n+1, k+1,1)-R B I B D$ and a $N R(n, k+1, k)-B I B D$, then there is $a((k+1) n, k+1, k)-R B I B D$.

Proof. Let $V_{1}=\left\{x_{i 1}, x_{i 2}, \ldots, x_{i k} \mid i=1,2, \ldots, n\right\}$ and let $V_{2}=\left\{y_{1}, y_{2}, \ldots\right.$, $\left.y_{n}\right\}$.

Let $G^{\prime}$ be a $G B T D(n, k)$ defined on $V_{1}$. Suppose the deficient $k$-tuple of row $i$ in $G^{\prime}$ is $\left\{x_{i 1}, x_{i 2}, \ldots, x_{i k}\right\}$ for $i=1,2, \ldots, n$. Let $D^{\prime}$ be a resolvable $(k n+1, k+1,1)-B I B D$ defined on $V \cup\{\infty\}$ so that the blocks containing $\infty$ are $\left\{\infty, x_{i 1}, x_{i 2}, \ldots, x_{i k}\right\}$ for $i=1,2, \ldots, n$. Let $D_{i}^{\prime}$ be the resolution class of $D$ which contains the block $\left\{\infty, x_{i 1}, x_{i 2}, \ldots, x_{i k}\right\}$ for $i=1,2, \ldots, n$. $N$ will denote a NR $(n, k+1, k)-B I B D$ defined on $V_{2}$ and $N_{i}$ will denote the resolution class of $N$ which does not contain the element $y_{i}$. 
We construct a resolvable $(k n+n, k+1, k)-B I B D$ on $V_{1} \cup V_{2}$ as follows. To each block in row $i$ of $G^{\prime}$ add the element $y_{i}(i=1,2, \ldots, n)$. Denote the resulting array of blocks of size $k+1$ by $G$. Let $C_{1}, C_{2}, \ldots, C_{n k-1}$ be the columns of $G$. Replace each block $\left\{\infty, x_{i 1}, x_{i 2}, \ldots, x_{i k}\right\}$ in $D^{\prime}$ with the block $\left\{y_{i}, x_{i 1}, x_{i 2}, \ldots, x_{i k}\right\}$ for $i=1,2, \ldots, n$. $D$ will denote the resulting configuration. Let $D_{i}$ be the corresponding resolution class of $D$ which contains the block $\left\{y_{i}, x_{i 1}, x_{i 2}, \ldots, x_{i k}\right\} \quad(i=1,2, \ldots, n)$.

The blocks in $G \cup D \cup N$ form a $(k n+n, k+1, k)-B I B D$. Every distinct pair in $V_{1}$ occurs $k-1$ times in $G$ and once in $D$. Every pair $\left\{y_{i}, x_{j \ell}\right\}$ occurs $k$ times in $G \cup D$. Every pair in $V_{2}$ occurs $k$ times in $N$. It is easy to verify that $\left\{C_{1}, C_{2}, \ldots, C_{n k-1}, D_{1} \cup N_{1}, D_{2} \cup N_{2}, \ldots, D_{n} \cup N_{n}\right\}$ is a resolution for this $(k n+n, k+1, k)-B I B D$ defined on $V_{1} \cup V_{2}$.

The following example illustrates this theorem. Since there exists a $G B T D(5,3)$ [19], a $(16,4,1)-R B I B D$ [7] and a $N R(5,4,3)-B I B D$, we can construct a $(20,4,3)-R B I B D$.

Theorem 5.2. If there exists a $G B T D(n, k)$, a $R G D D_{n-1}(n k ; k+1 ; k ; 0,1)$ and an $(n, k+1, k)-R B I B D$, then there is $a(k n+n, k+1, k)-R B I B D$.

Proof. Let $V_{1}=\left\{x_{i 1}, x_{i 2}, \ldots, x_{i k} \mid i=1,2, \ldots, n\right\}$ and let $V_{2}=\left\{y_{1}, y_{2}, \ldots\right.$, $\left.y_{n}\right\}$.

Let $G^{\prime}$ be a $\operatorname{GBTD}(n, k)$ defined on $V_{1}$. Suppose the deficient $k$-tuple of row $i$ in $G^{\prime}$ is $\left\{x_{i 1}, x_{i 2}, \ldots, x_{i k}\right\}$ for $i=1,2, \ldots, n$. Let $D$ be a $R G D D_{n-1}(n k ; k+1 ; k ; 0,1)$ defined on $V_{1}$ so that the groups of $D$ are the $k$-tuples $\left\{x_{i 1}, x_{i 2}, \ldots, x_{i k}\right\}$ for $i=1,2, \ldots, n$. Let $D_{1}, D_{2}, \ldots, D_{n-1}$ be the resolution classes of $D$. Let $N$ denote an $(n, k+1, k)-R B I B D$. $N_{1}, N_{2}, \ldots, N_{n-1}$ will be the resolution classes of $N$.

We construct a $(k n+n, k+1, k)-R B I B D$ as follows. To each block in row $i$ of $G^{\prime}$ add the elements $y_{i}(i=1,2, \ldots, n)$. Denote the resulting array of blocks of size $k+1$ by $G$. Let $C_{1}, C_{2}, \ldots, C_{n k-1}$ be the columns of $G$. Let $C_{n k}=\left\{\left\{y_{i}, x_{i 1}, x_{i 2}, \ldots, x_{i k}\right\} \mid i=1,2, \ldots, n\right\}$. The blocks in $G \cup D \cup N \cup C_{n k}$ form a $(k n+n, k+1, k)-B I B D$. Every distinct pair in $V_{1}$ occurs $k-1$ times in $G$ and once in $D \cup C_{n k}$. Every pair $\left\{y_{i}, x_{j \ell}\right\}$ occurs $k$ times in $G \cup C_{n k}$. Every pair in $V_{2}$ occurs $k$ times in $N$. It is easy to verify that $\left\{C_{1}, C_{2}, \ldots, C_{n k}, D_{1} \cup N_{1}, D_{2} \cup N_{2}, \ldots, D_{n-1} \cup N_{n-1}\right\}$ is a resolution for this $(k n+k, k+1, k)-B I B D$ defined on $V_{1} \cup V_{2}$.

The following is an example of this construction. Since a $\operatorname{GBTD}(8,3)$ exists [11], a $R G D D_{7}(24 ; 4 ; 3 ; 0,1)$ exists [34] and an $(8,4,3)-R B I B D$ is easily constructed, we can use Theorem 5.2 to construct a $(32,4,3)-R B I B D$.

Theorem 5.3. If there is a NGBTD $(n, k), a(k n+1, k+1,1)-R B I B D$ and $a$ $N R(n, k+1, k)-B I B D$, then there is a NR(kn+n+1,k+1,k)-BIBD.

Proof. Let $V_{1}=\left\{x_{1}, x_{2}, \ldots, x_{n k+1}\right\}$ and let $V_{2}=\left\{y_{1}, y_{2}, \ldots, y_{n}\right\}$.

Let $G^{\prime}$ be a $\operatorname{NGBTD}(n, k)$ defined on $V_{1}$. To each block in row $i$ of $G^{\prime}$ 
add the element $y_{i}, i=1,2, \ldots, n$. Denote the resulting array of blocks of size $k+1$ by $G$ and label the columns of $G, G_{1}, G_{2}, \ldots, G_{n k+1}$. Suppose the columns are arranged so that $G_{i}$ contains every element of $V_{1} \cup V_{2}-\left\{x_{i}\right\}$ precisely once.

Let $D$ be a $(k n+1, k+1,1)-R B I B D$ defined on $V_{1}$. Let $D_{1}, D_{2}, \ldots, D_{n}$ be a set of resolution classes for $D$. $N$ will denote a $N R(n, k+1, k)-B I B D$ defined on $V_{2}$ and $N_{i}$ will denote the resolution class of $N$ which does not contain $y_{i}$.

The blocks in $G \cup D \cup N$ form a $(k n+n+1, k+1, k)-B I B D$. Each pair of distinct elements from $V_{1}$ occurs $k-1$ times in $G$ and once in $D$. Each pair of distinct elements from $V_{2}$ occurs $k$ times in $N$. Finally, each pair of the form $\left\{x_{i}, y_{j}\right\}, \quad 1 \leq i \leq n k+1$ and $1 \leq j \leq n$, occurs $k$ times in $G$. A near resolution of this design is given by the following (near) resolution classes: $G_{1}, G_{2}, \ldots, G_{n k+1}, D_{1} \cup N_{1}, D_{2} \cup N_{2}, \ldots, D_{n} \cup N_{n}$.

A simple example of this construction is as follows. There exists a $N G B T D(5,3)$ [11], a $(16,4,1)-R B I B D$ [7] and a $N R(5,4,3)-B I B D$. We can use Theorem 5.3 to construct a $N R(21,4,3)-B I B D$.

In [19] we used $B T D S$ and $O B T D s$ to construct doubly resolvable and doubly near resolvable $B I B D s$ with block size 3 . We can generalize these constructions to use $G B T D s$ and $N G B T D s$ with block size $k$ to produce doubly resolvable and doubly near resolvable $B I B D s$ with block size $k+1$. In order to describe these constructions, we require several definitions.

Let $G$ be a $\operatorname{NGBTD}(n, k)$. Let $R_{1}, R_{2}, \ldots, R_{n}$ be the rows of $G$ and let $G_{1}, G_{2}, \ldots G_{n k+1}$ be the columns of $G . G^{\prime}=\left\{G_{1}, G_{2}, \ldots, G_{n k+1}\right\}$ is a (near) resolution of the underlying $(k n+1, k, k-1)-B I B D$. A resolution $D$, $D=\left\{D_{1}, D_{2}, \ldots, D_{k n}\right\}$, is called an orthogonal resolution to $G^{\prime}$ if

(i) $\left|G_{i} \cap D_{j}\right| \leq 1$ for $1 \leq i, j \leq k n+1$ and

(ii) $\left|D_{j} \cap R_{i}\right|=1$ for $1 \leq j \leq k n+1,1 \leq i \leq n$.

If $D$ exists, we say that the $\operatorname{NGBTD}(n, k)$ has a pair of orthogonal resolutions $(O R S)$.

For $G B T D s$, as in the case of $B T D s$, we define a pair of almost orthogonal resolutions. Let $G$ be a $G B T D(n, k)$. Let $R_{1}, R_{2}, \ldots, R_{n}$ be the rows of $G$ and let $G_{1}, G_{2}, \ldots G_{k n-1}$ be the columns of $G . G^{\prime}=\left\{G_{1}, G_{2}, \ldots, G_{k n-1}\right\}$ is a resolution of the underlying $(k n, k, k-1)-B I B D$. A resolution $D=$ $\left\{D_{1}, D_{2}, \ldots, D_{k n-1}\right\}$ will be called almost orthogonal to $G^{\prime}$ if

(i) $G_{k n-1}=D_{k n-1}$,

(ii) $\left|G_{i} \cap D_{j}\right| \leq 1$ for $1 \leq i, j \leq k n-2$,

(iii) $\left|D_{j} \cap R_{i}\right|=1$ for $1 \leq j \leq k n-2,1 \leq i \leq n$.

If $D$ exists, we say that $G$ has a pair of almost orthogonal resolutions (denoted by $A O R s)$. If $G$ is a $\operatorname{GBT} D(n, k)$ with a pair of almost orthogonal resolutions with the property that the deficient $k$-tuples of $G$ are contained in the shared 
resolution class $G_{k n-1}$, then we say that $G$ has property $C^{\prime}$.

Our constructions will also require the existence of $K S_{k+1}(k n+1 ; 1,1) s$ with complementary $(1, k ; k+1, n, 1)$-frames.

Let $K$ be a $K S_{k+1}(k n+1 ; 1,1)$ defined on $V \cup\{\infty\}$ where $\infty$ occurs in each cell of the main diagonal.

We say $K$ has a complementary $(1, k ; k+1, n, 1)$-frame (or a complementary $D N R(n, k+1, k)-B I B D)$ if there exists a $(1, k ; k+1, n, 1)$-frame (or a $D N R(n, k+1, k)-B I B D)$ which can be written in the empty cells of $K$.

Theorem 5.4. If there is a $\operatorname{GBTD}(n, k)$ with a pair of almost orthogonal resolutions and Property $C^{\prime}$ and if there is a $K S_{k+1}(k n+1 ; 1,1)$ with a complementary $(1, k ; k+1, n, 1)$-frame, then there is a $K S_{k+1}(k n+n+1 ; 1, k)$ or a $D R(k n+n+1, k+1, k)-B I B D$.

Proof. Let $V_{i}=\left\{x_{i 1}, x_{i 2}, \ldots, x_{i k}\right\}$ for $i=1,2, \ldots, n$ and let $V=\bigcup_{i=1}^{n} V_{i}$. Let $W=\left\{y_{1}, y_{2}, \ldots, y_{n}\right\}$.

Let $G^{\prime}$ be a $G B T D(n, k)$ defined on $V$ with a pair of almost orthogonal resolutions and Property $C^{\prime}$. Suppose the deficient $k$-tuples of $G^{\prime}$ are $V_{i}$ for $i=1,2, \ldots, n$. Let $C=\left(C_{1}, C_{2}, \ldots, C_{n}\right)^{T}$ be the column of deficient $k$ tuples where $C_{i}$ is the deficient $k$-tuple for row $i$. Since $G^{\prime}$ has Property $C^{\prime}, V_{i}=C_{j}$ for some $j$. Denote the columns of $G^{\prime}$ by $A_{1}^{\prime}, A_{2}^{\prime}, \ldots, A_{k n+1}^{\prime}$. Let $D=\left\{D_{1}^{\prime}, \ldots, D_{k n-1}^{\prime}\right\}$ be an almost orthogonal resolution for $G^{\prime}$. Add a new element $y_{i}$ to each $k$-tuple in row $i$ of $G^{\prime}, \quad 1 \leq i \leq n$, and call the resulting array $G$. We label the first $k n-2$ columns of $G, A_{1}, A_{2}, \ldots, A_{k n-2}$, and the last column of $G$ we label $X$. ( $X_{i}$ denotes the block in row $i$ of $X$.) The almost orthogonal resolution $D^{\prime}$ provides an almost orthogonal resolution $D$ in $G$. Let $D=\left\{D_{1}, D_{2}, \ldots, D_{k n-2}, X\right\}$.

Let $K$ be a $K S_{k n}(k n+1 ; 1,1)$ defined on $V \cup\{\infty\}$ such that the main diagonal of $K$ contains the $(k+1)$-tuple $C_{i} \cup\{\infty\}$ in cell $(i, i)$ for $i=$ $1,2, \ldots, n$. Replace the $(k+1)$-tuple $C_{i} \cup\{\infty\}$ with the $(k+1)$-tuple $C_{i} \cup\left\{y_{i}\right\}$ for $i=1,2, \ldots, n$. Let $Y_{i}=C_{i} \cup\left\{y_{i}\right\}$ and let $Y=\left\{Y_{1}, Y_{2}, \ldots, Y_{n}\right\}$. Let $F$ be a complementary $(1, k ; k+1, n, 1)$-frame defined on $W$ where row $i$ and column $i$ of $F$ do not contain the element $y_{i}, 1 \leq i \leq n$. Place $F$ in the empty cells of $K$ and delete the main diagonal of this new array of $(k+1)$ tuples. Call the resulting array $E$. Label the rows of $E$ by $T_{1}, T_{2}, \ldots, T_{n}$ and the columns of $E$ by $S_{1}, S_{2}, \ldots, S_{n}$.

It is straightforward to verify that the collection of $(k+1)$-tuples in $G \cup E \cup Y$ forms a $(k n+n, k+1, k)-B I B D$. We list a pair of resolutions $R^{1}$ and $R^{2}$ for the design.

$$
\begin{aligned}
& R^{1}=\left\{X, A_{1}, A_{2}, \ldots, A_{k n-2}, T_{1} \cup Y_{1}, T_{2} \cup Y_{2}, \ldots, T_{n} \cup Y_{n}\right\}, \\
& R^{2}=\left\{Y, D_{1}, D_{2}, \ldots, D_{k n-2}, S_{1} \cup X_{1}, S_{2} \cup X_{2}, \ldots, S_{n} \cup X_{n}\right\} .
\end{aligned}
$$

We have a similar result which uses NGBTDs.

Theorem 5.5. If there is a $\operatorname{NGBTD}(n, k)$ with a pair of orthogonal resolutions 
and a $K S_{k+1}(k n+1 ; 1,1)$ with a complementary $(1, k ; k+1, n, 1)$-frame (or a complementary $D N R(n, k+1, k)-B I B D)$, then there is a $D N R(n k+$ $n+1, k+1, k)-B I B D$.

Proof. Let $V_{1}=\left\{x_{1}, x_{2}, \ldots, x_{n k+1}\right\}$ and let $V_{2}=\left\{y_{1}, y_{2}, \ldots, y_{n}\right\}$. Let $G^{\prime}$ be a $N G B T D(n, k)$ defined on $V_{1}$. Suppose column $i$ of $G^{\prime}$ does not contain the element $x_{i}$ for $i=1,2, \ldots, k n+1$. To each $k$-tuple in row $i$ of $G^{\prime}$ add the element $y_{i}, 1 \leq i \leq n$. Let $G$ denote the resulting array of blocks of size $k+1$. The columns of $G$ will be denoted by $G_{1}, G_{2}, \ldots, G_{k n+1} \cdot G_{i}$ contains every element of $\left(V_{1}-\left\{x_{i}\right\}\right) \cup V_{2}$ precisely once. The orthogonal resolution for $G^{\prime}$ will partition the blocks of $G$ into $k n+1$ (near) resolution classes; call these $D_{1}, D_{2}, \ldots, D_{k n+1}$. Suppose $D_{i}$ contains every element of $\left(V_{1}-\left\{x_{i}\right\}\right) \cup V_{2}$ precisely once.

Let $K^{\prime}$ be a $K S_{k+1}(k n+1 ; 1,1)$ defined on $V_{1}$. Place a complementary $(1, k ; k+1, n, 1)$-frame (or a complementary $D N R(n, k+1, k)-B I B D$ ) defined on $V_{2}$ in the empty cells of $K^{\prime}$. Let $K$ denote the resulting array of $(k+1)$-tuples. Let $T_{1}, T_{2}, \ldots, T_{n}$ denote the rows of $K$ and let $S_{1}, S_{2}, \ldots, S_{n}$ denote the columns of $K$. Suppose $T_{i}$ contains every element of $V_{1} \cup\left(V_{2}-\left\{y_{i}\right\}\right)$ precisely once and suppose $S_{i}$ contains every element of $V_{1} \cup\left(V_{2}-\left\{y_{i}^{\prime}\right\}\right)$ precisely once $(i=1,2, \ldots, n)$. (If we have used a complementary $(1, k ; k+1, n, 1)$-frame, then $y_{i}=y_{i}^{\prime}$.)

The collection of blocks in $K \cup G$ forms a $(k n+n+1, k+1, k)-B I B D$. One (near) resolution of this design consists of $\left\{G_{1}, G_{2}, \ldots, G_{k n+1}, T_{1}, T_{2}, \ldots\right.$, $\left.T_{n}\right\}$. An orthogonal resolution is given by

$$
\left\{D_{1}, D_{2}, \ldots, D_{k n}, S_{1}, S_{2}, \ldots, S_{n}\right\}
$$

Existence results using the constructions in Theorems 5.4 and 5.5 for block size $k=2$ appear in [9]. Thus far, we have not constructed any examples for $k \geq 3$. Very little is known about the Kirkman squares, $K S_{k+1}(k n+1 ; 1,1)$, or the $(1, k ; k+1, n, 1)$-frames which are required for $k \geq 3$.

For example, the smallest $K S_{4}(v ; 1,1)$ known to exist is $v=64$ [5]. In order to apply our constructions, we would need to find a complementary $(1,3 ; 4,21,1)$-frame.

\section{SUMMARY}

We have introduced generalized balanced tournament designs and shown that these designs are related to other types of combinatorial designs. We have also described how to use $G B T D S$ to construct resolvable, near resolvable and doubly resolvable $B I B D s$. These constructions together with our existence results for $G B T D$ s have already produced several new classes of designs. These are described in $[10,11,12]$. 


\section{REFERENCES}

1. R. D. Baker, Resolvable BIBDs and SOLS, Discrete Math. 44 (1983), 13-29.

2. Demeng Chu and Lie Zhu, Existence of resolvable balanced incomplete block designs with $k=5$ and $\lambda=1$, Ars Combin. 24 (1987), 185-192.

3. D. G. Curran and S. A. Vanstone, Doubly resolvable designs from generalized Bhaskar Rao designs, Discrete Math. 73 (1988/89), 49-63.

4. J. H. Dinitz and D. R. Stinson, The construction and uses of frames, Ars Combin. 10 (1980), 31-53.

5. R. Fuji-Hara and S. A. Vanstone, On the spectrum of doubly resolvable designs, Congr. Numer. 28 (1980), 399-407.

6. H. Hanani, On resolvable balanced incomplete block designs, J. Combin. Theory Ser. A 17 (1974), 275-289.

7. H. Hanani, D. K. Ray-Chaudhuri and R. M. Wilson, On resolvable designs, Discrete Math. 3 (1972), 343-357.

8. E. R. Lamken, A note on partitioned balanced tournament designs, Ars Combin. 24 (1987), $5-16$

9. __ On classes of doubly resolvable ( $v, 3,2)-B I B D$ s from balanced tournament designs, Ars Combin. 24 (1987), 85-91.

10. _ Constructions for generalized balanced tournament designs, (submitted).

11. __ Existence results for generalized balanced tournament designs with block size 3 , in preparation.

12. - The existence of doubly resolvable $(v, 3,2)-B I B D s$, in preparation.

13. __ 3-complementary frames and doubly near resolvable $(v, 3,2)-B I B D s$, Discrete Math. (to appear).

14. __ Coverings, orthogonally resolvable designs and related combinatorial configurations, Ph.D Thesis, University of Michigan, 1983.

15. E. R. Lamken and S. A. Vanstone, The existence of factored balanced tournament designs, Ars Combin. 19 (1985), 157-160.

16. $\ldots$ The existence of partitioned balanced tournament designs of side $4 n+1$, Ars Combin. 20 (1985), 29-44.

17. _ The existence of partitioned balanced tournament designs of side $4 n+3$, Ann. Discrete Math. 34 (1987), 319-338.

18. _ The existence of partitioned balanced tournament designs, Ann. Discrete Math. 34 (1987), 339-352.

19. __ Balanced tournament designs and resolvable $(v, 3,2)-B I B D s$, Discrete Math. (to appear).

20. __ Balanced tournament design and related topics, Ann. Discrete Math. (to appear).

21. __ Orthogonal resolutions in odd balanced tournament designs, Graphs and Combinatorics 4 (1988), 241-255.

22. __ Balanced tournament designs with almost orthogonal resolutions, J. Austral. Math. Soc. (Ser. A) (to appear).

23. _ The existence of a class of Kirkman squares of index 2, J. Austral. Math. Soc. (Ser. A) 44 (1988), 33-41.

24. __ Existence results for doubly near resolvable ( $v, 3,2)-B I B D s$, (submitted).

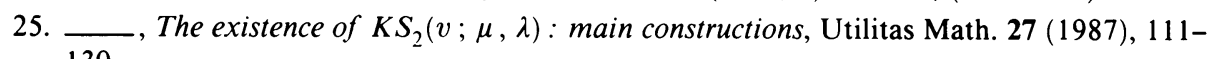
130.

26. $\ldots$ The existence of $K S_{2}(v ; \mu, \lambda)$ : special constructions, Utilitas Math. 27 (1985), 131-155.

27. R. C. Mullin and W. D. Wallis, The existence of Room squares, Aequationes Math. 1 (1975), 1-7. 
28. D. K. Ray-Chaudhuri and R. M. Wilson, Solution of Kirkman's school girl problem, Proc. Sympos. Pure Math., vol. 19, Amer. Math. Soc., Providence, R.I., 1971, pp. 187-203.

29. R. Rees and D. R. Stinson, On resolvable group divisible designs with block size 3, Ars Combin. 23 (1987), 107-120.

30. A. Rosa and S. A. Vanstone, Starter-adder techniques for Kirkman squares and Kirkman cubes of small sides, Ars Combin. 14 (1982), 199-212.

31. B. Rokowska, Resolvable systems of 8-tuples, J. Statist. Planning Info. 9 (1984), 131-141.

32. P. J. Schellenberg, G. H. J. Van Rees and S. A. Vanstone, The existence of balanced tournament designs, Ars Combin. 3 (1977), 303-318.

33. P. Smith, A doubly divisible nearly Kirkman system, Discrete Math. 18 (1977), 93-96.

34. D. R. Stinson, Room squares with maximum empty subarrays, Ars Combin. 20 (1985), 159-166.

35. _ _ Frames for Kirkman triple systems, Discrete Math. 65 (1987), 289-300.

36. $\ldots$, The equivalence of certain incomplete transversal designs and frames, Ars Combin. 22 (1986), 81-87.

37. Ph.D. Thesis, University of Waterloo, 1981.

38. S. A. Vanstone, Doubly resolvable designs, Discrete Math. 29 (1980), 77-86.

39. _ On mutually orthogonal resolutions and near resolutions, Ann. Discrete Math. 15 (1982), 357-369.

Institute for Mathematics and its Applications, University of Minnesota, MinneapoLIS, MiNNESOTA 55455

Current address: Center for Communications Research, Institute for Defense Analyses, Princeton, New Jersey 08540 